\title{
PREVALENCIA DE DISLIPIDEMIA EN ARTRITIS TEMPRANA Y SU RELACIÓN CON ACTIVIDAD INFLAMATORIA
}

Lipid profile in early arthritis and its relation with inflammatory activity

\author{
Asbert, $P$, Gobbi ${ }^{1}, C$; Alba, $P^{1}$; Alessio, $D^{1}$; Subils, $G^{1}$; Dotto $G^{1}$; Demarchi, $M^{1}$; Albiero, $E^{1}$; Salica, $D^{1}$; \\ Martínez, $F^{1}$
}

\section{Resumen:}

El riesgo cardiovascular está aumentado en pacientes con Artritis Reumatoidea establecida, con aumento de la morbimortalidad cardiovascular, inclusive tempranamente y puede ser expresión subclínica de la enfermedad. Pocos estudios han evaluado el perfil lipídico en artritis temprana (AT). OBJETIVOS: Determinar la prevalencia y el tipo de alteración del perfil lipídico en pacientes con AT y su asociación con la actividad de la enfermedad (AE). MATERIAL Y MÉTODOS: Se estudiaron pacientes con diagnóstico de AT comparados con un grupo control, apareados por sexo, edad y factores de riesgo cardiovascular, asistidos de forma consecutiva desde enero de 2011 a mayo de 2013 en el Hospital Córdoba. Se recolectaron datos demográficos, perfil lipídico y actividad de la enfermedad (AE) por Disease Activity Score (DAS 28). Se comparó el nivel de lípidos en ambos grupos y entre pacientes con artritis según el nivel de $A E$, clasificándolos en Baja AE y Mediana / Alta AE. $P$ menor a 0.05 fue considerada significativa. RESULTADOS: El número de pacientes con Artritis temprana fue de 31 , con una media de edad de 42.3 años, $87 \%$ sexo femenino y el nivel de Colesterol Total promedio fue de $191.9 \mathrm{mg} / \mathrm{dl}$, HDL 54, LDL 115.8, Triglicéridos 117,6, mientras que el grupo control fue de 31 pacientes con una media de edad de 42.7 años, con nivel promedio de Colesterol 198.7 mg/dl, HDL 56.9, LDL 122.6, Triglicéridos $99.6 \quad(p=N S$, para todas las determinaciones). En cuanto a la actividad de la enfermedad, en el grupo de Baja AE los niveles de Colesterol fueron 196.3, LDL 115.8, HDL 62 y triglicéridos 95.17 y en el grupo moderada y alta

Palabras clave: artritis, dislipemias
AE, Colesterol 190,1 LDL 115, 8, HDL 52 y triglicéridos 122,9 (p NS para todas las comparaciones)

CONCLUSIONES: El perfil lipídico fue normal y no se encontró asociado a la $A E$ en pacientes con AT.

\section{Abstract:}

Cardiovascular risk is increased in Rheumatoid Arthritis and it is associated with higher morbility and mortality. Few studies have evaluated the lipid profile in early arthritis (EA).

Aims: To study the lipid profile in patients with EA and its association with disease activity (DA). Methods: We studied 31 patients with diagnosis of EA and a control group, with age, gender and cardiovascular risk factors matched, who were attended to Rheumatology Unit at Córdoba Hospital from January 2011 to May 2013. We evaluated demographic data, lipid profile and DA by DAS28. Results: 31 patients were included with mean age of 42.3 years old, $87 \%$ female, the cholesterol level was $191.9 \mathrm{mg} / \mathrm{dl}$, HDL 54, LDL 115.8, Triglycerides 117,6 ; and 31 patients were included in the control group with average age of 42.7 years old, and cholesterol level of $198.7 \mathrm{mg} / \mathrm{dl}$, HDL 56.9 LDL 122.6, Triglycerides 99.6 (p NS). Regards disease activity, in the low DA group the Cholesterol level was 196.3, LDL 115.8, HDL 62 y triglycerides 95.17, and in the Moderate and High DA the Cholesterol level was $190 \mathrm{mg} / \mathrm{dl}$, LDL 115, HDL 52 y triglycerides 122,9 ( $p$ NS)

CONCLUSIONS : The lipid profile was normal and it was not associated with DA in EA patients.

Key words: arthritis; dyslipidemias

1. Unidad de Reumatología de la Cátedra de Semiología, Cátedra de Clínica Médica I y Servicio de Laboratorio del Hospital Córdoba. Email: carlaandreagobbi@hotmail.com 


\section{Introducción}

La Artritis Reumatoidea (AR) es una enfermedad inflamatoria crónica que afecta al tejido conectivo, causando destrucción articular y discapacidad física ${ }^{1-4}$. Paralelamente, se describen otras manifestaciones extraarticulares que varían desde síntomas constitucionales hasta compromiso orgánico incluyendo a la Enfermedad Cardiovascular (ECV) ${ }^{1-5}$.

Los pacientes con AR presentan un aumento de la mortalidad comparado con la población general ${ }^{1,2,6-8}$, a pesar de los avances del tratamiento logrados en las últimas décadas. La ECV es la principal causa de mortalidad, en la mayoría de los estudios, tanto en AR establecida como temprana ${ }^{3,9-14}$.

Los factores de riesgo tradicionales para ateroesclerosis, incluidos edad, hipertensión, tabaquismo y desordenes del perfil lipídico contribuyen a la enfermedad cardiovascular de mala evolución ${ }^{2}, \quad 5,12-19$, sin embargo, no explican completamente el aumento de eventos cardiovasculares. ${ }^{20}$.

El riesgo cardiovascular se encuentra aumentado dentro de los 2 años del diagnóstico de AR, se piensa que la inflamación sistémica puede inducir ateroesclerosis acelerada, ${ }^{8,13,21-27}$, contribuir al riesgo de ECV a través de múltiples mecanismos, desde su efecto en la función endotelial hasta su efecto en el perfil lipídico ${ }^{29}$.

La dislipemia es altamente prevalente en AR afectando entre $55-65 \%$ de los pacientes ${ }^{29-31}$. Numerosos factores influyen en su desarrollo incluyendo la predisposición genética, actividad de la enfermedad, actividad física limitada por dolor o discapacidad, tratamiento instaurado ${ }^{29}$. Otros mecanismos incluyen los efectos de las citocinas en el tejido adiposo, liberando más ácidos grasos libres (AGL) en el hígado, incrementando la síntesis de AGL y triglicéridos; y en el endotelio vascular, reduciendo la actividad de la lipasa lipoproteína, principal enzima catabólica de los lípidos ricos en triglicéridos ${ }^{32}$.

El medio ambiente inflamatorio y los mecanismos antioxidantes alterados en AR pueden promover la oxidación de LDL, facilitando aterogénesis y aumentando el riesgo cardiovascular ${ }^{7}$, 33

El objetivo de este estudio fue determinar prevalencia de dislipemia y caracterizar el perfil lipídico en pacientes con Artritis Temprana (AT) atendidos en un hospital general y su asociación con actividad de la enfermedad.

\section{Material y métodos}

Se realizó un estudio corte transversal en pacientes con Artritis Temprana (AT), definiendo como tal el compromiso igual o mayor a 3 articulaciones y menor de un año de evolución, que realizaron consulta en la Unidad de Reumatología del Hospital Córdoba desde enero de 2011 a mayo de 2013

Los criterios de inclusión fueron: edad mayor de 15 años y diagnóstico de AT. El grupo control está compuesto por pacientes voluntarios sanos, sin Artritis Reumatoidea, de la misma zona geográfica, apareados por sexo, edad y factores de riesgo cardiovascular.

Se recolectaron datos demográficos y datos clínicos. La Hipertensión (HTA) fue definida acorde a la definición del Séptimo Reporte ${ }^{34}$, Diabetes Mellitus (DBT) acorde a los criterios de ADA ${ }^{35}$. La enfermedad Cardiovascular (ECV) fue definida por la presencia de Accidente Cerebrovascular $(A C V)^{36}$. y cardiopatía Isquémica $(C l)^{37}$. El Tabaquismo (TBQ) se evaluó acorde a las recomendaciones de SEPAR ${ }^{38}$, el diagnóstico de dislipemia (DLP) según los criterios del ATP $I I^{39}$.

En el examen físico se evaluó el recuento de articulaciones inflamadas y dolorosas, el peso y la talla y se determinó índice de masa corporal (IMC) acorde a la clasificación de la $\mathrm{OMS}^{40}$.

Los análisis de laboratorio realizados fueron: Colesterol total $(\mathrm{mg} / \mathrm{dl}), \mathrm{LDL}(\mathrm{mg} / \mathrm{dl}), \mathrm{HDL}(\mathrm{mg} / \mathrm{dl})$, triglicéridos, glucemia, VSG (acelerada mayor a $20 \mathrm{~mm} / \mathrm{h}$ ), anticuerpos anti-CCP, Factor Reumatoideo y anticuerpos antinucleares. 
La actividad de la enfermedad se determinó por el índice Disease Activity Score (DAS-28) ${ }^{41,42}$ Definiendo Alta Actividad de la enfermedad DAS mayor a 5,1; Moderada Actividad 3,2 a 5; Baja Actividad menor a 3,2; Remisión menor a 2,6.Se los agrupó en dos grupos: DAS 28 menor a 3,2 y mayor a 3,2.

\section{Análisis estadístico:}

Variables Continuas: Se analizó Media y Error Estándar (EE) para edad, IMC, Colesterol total, Triglicéridos, HDL, LDL, VSG, Anti CCP y DAS-28.

Variables Categóricas: Se analizó frecuencia relativa (porcentajes) para sexo, $\mathrm{FR}$, antecedentes de HTA, DBT, HTA, TBQ y ACV/IAM.

Para estadística inferencial se utilizó para las variables mensurables el test no paramétrico $U$ de Mann-Whitney, para las categóricas se utilizó Chi cuadrado. Se consideró como nivel de significación un valor de $p<0,05$.

\section{Resultados}

El número de pacientes incluidos fue de 31, 27 fueron mujeres (87\%) y 4 Hombres (13\%), con un promedio de edad de $42,32 \pm 2,68$. El factor reumatoide fue positivo en 17 pacientes (55\%), 25 tenían anti-CCP positivo (81\%). EI DAS 28 medio fue de 5,05 $\pm 0,33$. Las características clínicas de los pacientes se muestran en Tabla 1.

Los resultados del perfil lipídico de los pacientes y controles se muestran en Tabla 2

La relación de Perfil lipídico con la actividad de la enfermedad y el nivel de VSG en tablas 3 y 4.

Tabla 1: Características Generales de la Población

\begin{tabular}{|l|l|}
\hline Caracteristicas & Valor \\
\hline $\mathrm{N}^{\circ}$ de pacientes & 31 \\
\hline Sexo femenino/Sexo masculino & $27 / 4$ \\
\hline Edad & $42,32 \pm 2,68(16-61)$ \\
\hline FR + & $55 \%(17)$ \\
\hline AntiCCP + & $81 \%(25)$ \\
\hline $\begin{array}{l}\text { Anticuerpos antinucleares (mayor } \\
\text { 1/160) }\end{array}$ & $25 \%(8)$ \\
\hline DBT & $6 \%$ \\
\hline HTA & $86 \%$ \\
\hline Dislipemia & $25 \%$ \\
\hline TBQ & $29 \%$ \\
\hline ECV (IAM/ACV) & $3 \%$ \\
\hline IMC & $26,06 \pm 0,94(18,33-36.78)$ \\
\hline
\end{tabular}

Tabla 2 : Perfil Lipidico en pacientes y controles

\begin{tabular}{|c|c|c|c|c|}
\hline & CT & HDL & LDL & TGS \\
\hline AT & $\begin{array}{l}191,9 \\
\mathrm{mg} / \mathrm{dl} \pm 9,55\end{array}$ & $\begin{array}{l}54 \\
\mathrm{mg} / \mathrm{dl} \pm 2,44\end{array}$ & $\begin{array}{l}115,8 \\
\mathrm{mg} / \mathrm{dl} \pm 6,99\end{array}$ & $\begin{array}{l}117,6 \\
\mathrm{mg} / \mathrm{dl} \pm 11,92\end{array}$ \\
\hline Control & $198,7 \mathrm{mg} / \mathrm{dl} \pm 7,66$ & $\begin{array}{l}56,9 \\
\mathrm{mg} / \mathrm{dl} \pm 2,56\end{array}$ & $\begin{array}{l}122,6 \\
\mathrm{mg} / \mathrm{dl} \pm 6,86\end{array}$ & $\begin{array}{l}99,6 \\
\mathrm{mg} / \mathrm{dl} \pm 9,45\end{array}$ \\
\hline$p$ & 0,21 & 0,23 & 0,23 & 0,18 \\
\hline
\end{tabular}


Tabla N ${ }^{\circ} 3$ Relacion Perfil Lipidico con Actividad de enfermedad (DAS 28)

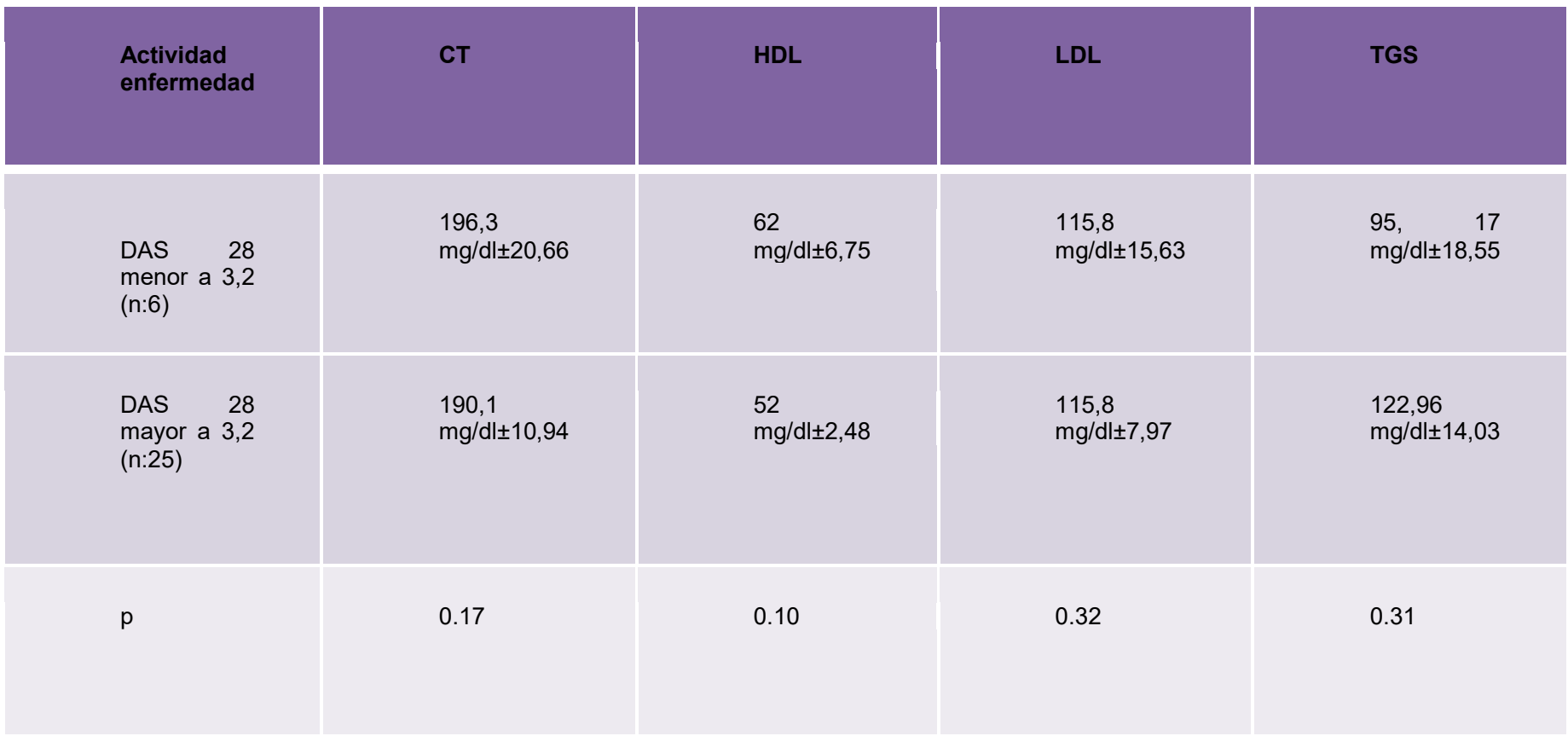

Tabla: 4 Perfil lipidico en Artritis con VSG alterada y normal

\begin{tabular}{|l|l|l|l|}
\hline & $\begin{array}{l}\text { Colesterol } \\
\text { Total }\end{array}$ & Triglicéridos & HDL \\
\hline $\begin{array}{l}\text { Artritis VSG } \\
\text { alterada (n: 18) }\end{array}$ & $188,56 \pm 15,26$ & $106,31 \pm 13,55$ & $50,50 \pm 2,98$ \\
\hline $\begin{array}{l}\text { Artritis VSG } \\
\text { normal (n: 13) }\end{array}$ & $196,54 \pm 9,15$ & $132,77 \pm 21,31$ & $58,85 \pm 3,82$ \\
\hline
\end{tabular}

El riesgo de dislipemia no está aumentado por actividad de enfermedad, medida por DAS 28 (OR 1,05)

El riesgo de disminución de HDL esta aumentado en 2,83 veces $(0,47-17,23)$ cuando actividad de enfermedad es moderada a alta ( $P=N S)$

El riesgo de padecer dislipemia es 1,57 veces $(0,28-8,85)$ mayor en los casos que tienen VSG alterada $(0,11-3,59)$ comparado con los que presentan VSG normal ( $P=N S$ ). El riesgo de disminución de HDL en casos con VSG elevada es 1,8 mayor $(0,43-7,6)$ que los que tienen VSG normal. ( $P=N S$ )

\section{Discusión}

El estudio del perfil lipídico en pacientes con $A R$ ha tenido resultados contradictorios. Numerosos ensayos han observado discrepancias en los resultados que puede obedecer a la diferencia en las poblaciones seleccionadas, tipo de estudio, tipo de enfermedad o actividad de la enfermedad ${ }^{25,43-47}$

En nuestro estudio sobre artritis temprana no encontramos alteración del perfil lipídico comparado con la población control, esto contrasta con lo que se demostró en enfermedad establecida.

Se ha demostrado que el perfil lipídico de pacientes con AR activa sin tratamiento exhibe un perfil aterogénico caracterizado por aumento de CT, LDL, no HDL y TGS y disminución sérica de HDL, siendo este último el hallazgo más consistente $2,25,43,44,48-51$, sin embargo en algunos estudios no muestran alteraciones ${ }^{52-54}$. Se objetiva esta alteración del perfil aterogénico mayoritariamente en AR establecida activa y no tratada a diferencia de población general ${ }^{25,44,50}$. En el estudio QUEST-RA, se observó una relación entre la hiperlipidemia e infarto agudo de miocardio, sugiriendo la influencia de AR y factores de riesgo tradicionales para el desarrollo de 
eventos cardiovasculares, también hubo disminución del riesgo cardiovascular con el tratamiento de la enfermedad ${ }^{55}$.

El estudio NHANES III comparó niveles lipídico entre AR y no AR en pacientes mayores de 60 años. Se examinó también un subgrupo con actividad más severa. Mostró niveles significativamente menores de HDL colesterol en pacientes con AR, pero a pesar de que el perfil lipídico fue menos favorable en la cohorte de AR ningún parámetro fue estadísticamente significativo. Las anomalías lipoproteicas en pacientes AR sin tratamiento, son similares a los vistos en pacientes con Síndrome Metabólico, con HDL bajo y aumento de $\mathrm{TGS}^{54}$. En varios estudios los niveles de HDL se correlacionaron negativamente con PCR ${ }^{43,54}$.

Se realizaron esfuerzos para investigar la fase preclínica, sin embargo los resultados son contradictorios y no coinciden con nuestro estudio sobre artritis temprana. Van Halm ${ }^{56}$ observo que el perfil lipídico de los donantes que desarrollaron AR a posteriori es más aterogénico que los controles apareados, caracterizado por elevación de CT, TGS y APO B y HDL disminuida, este fenómeno comenzó 10 años antes de fase clínica ${ }^{56}$. En cambio en el estudio de Myasosediva $^{57}$ se observó una disminución significativa en los niveles de CT y LDL durante los 5 años antes de la incidencia de la AR en comparación con sujetos no-RA ${ }^{57}$.

La inflamación activa debido a AR ha sido sugerida como un factor que disminuye los niveles de HDL y posiblemente los niveles de colesterol total, y que mejorarían luego del control de la enfermedad. Las medidas de colesterol en pacientes con AR en periodos de inflamación activa pueden no reflejar los niveles basales ${ }^{2}$.

Pocos estudios han evaluado los niveles lipídico y su asociación con la actividad de la enfermedad. Svenson observó en un estudio de corte trasversal en 28 pacientes con AR, con una media de duración de la enfermedad de 7 años, una relación inversamente proporcional entre actividad de la enfermedad y el perfil lipídico ${ }^{58}$, estos hallazgos se confirmaron por White con un número mayor de pacientes con AR que demostró una relación inversa entre la elevación de PCR y VSG y los niveles de $\mathrm{HDL}^{59}$. Nuestro estudio presenta una limitación importante en el número de pacientes, sin embargo, a pesar de que no hubo significación estadística, el OR sugiere que HDL podrían disminuir tempranamente debido a actividad de la enfermedad medida por DAS y aumento de la VSG, como así también el riesgo de dislipemia cuando la VSG está acelerada, esto debería confirmarse con mayor número de casos.

\section{Conclusiones}

A pesar de que el perfil lipídico fue normal y no se encontró asociado a la $A E$ en pacientes con AT, el OR muestra mayor riesgo de que el HDL disminuye en los pacientes con artritis activa, medida por DAS 28 , o con VSG acelerada. Son necesarios estudios de mayor envergadura para confirmar estos hallazgos.

No existe conflicto de interés entre los autores.

Este estudio se realizó con subsidio Secyt. 


\section{Bibliografía}

1. Toms T, Panoulas V., Kitas G. Dyslipidaemia in Rheumatological Autoimmune Diseases The Open Cardiovascular Medicine Journal, 2011, 5, 64-75 1874$1924 / 112011$

2. Van Doornum S, McColl G, Wicks I. Accelerated Atherosclerosis An Extraarticular Feature of Rheumatoid Arthritis? ARTHRITIS \& RHEUMATISM. 2002;46(4): 862873

3. Sandoo A, Veldhuijzen van Zanten J, Metsios G, Carroll D, Kitas G. Vascular function and morphology in rheumatoid arthritis: a systematic review. Rheumatology. 2011; 50:2125-2139.

4. Harris E. Clinical features of rheumatoid arthritis. In: Harris E, Budd R, Firestein G, Genovese M, Sergent J, Ruddy S ,et al, editors Kelley's textbook of rheumatology.7th ed.Philadelphia: Elsevier Saunders; 2005:1243-78

5. Nurmohamed MT. Atherogenic lipid profile and its management in patients with rheumatoid arthritis. Vasc Health Risk Manag 2007; 3(6):845-852

6. Turesson C, O'Fallon W, Crowson C, Gabriel S, Matteson E. Occurrence

extraarticulardiseasemanifestationsisassociatedwithexcessm ortality in a community based cohort of patients with rheumatoid arthritis. JRheumatol. 2002; 29:62-7

7. Nakken B, Szodoray P. Accelerated atherosclerosis in rheumatoid arthritis: rationale for mannose- binding lectins. J Rheumatol 2010; 37(3):482-484.

8. Maradit-Kremers $\mathrm{H}$, Crowson $\mathrm{C}$, Nicola $\mathrm{P}$, Ballman $\mathrm{K}$, Roger $\mathrm{V}$, Jacobsen $\mathrm{S}$ et al. Increased unrecognized coronary heart disease and sudden deaths in rheumatoid arthritis: a population-based cohort study. Arthritis and Rheumatism. 2005; 52(2):402-411

9. Meune C, Touz'e E, Trinquart L, Allanore Y. High risk of clinical cardiovascular events in rheumatoid arthritis: levels of associations of myocardial infarction and stroke through a systematic review and meta-analysis. Archives of Cardiovascular Diseases. 2010; 103(4): 253-261

10. Cavagna L, Boffini N, Cagnotto G, Inverardi F, Grosso V, Caporali R. Atherosclerosis and Rheumatoid Arthritis: More Than a Simple Association. Mediators of Inflammation. 2012; 2012:1-9.

11. Naz S, Symmons D. Mortality in established rheumatoid arthritis. Best Pract Res ClinRheumatol 2007; 21:871-83

12. Roman M, Salmon J. Cardiovascular Manifestations of rheumatologic Diseases. Circulation. 2007; 116: 2346-2355

13. Solomon D, Goodson N, Katz J, Weinblatt M, Avorn J, Setoguchi S, et al. Patterns of cardiovascular risk in rheumatoid arthritis Ann Rheum Dis 2006; 65:1608-1612.

14. Rindfleisch J, Muller D. Diagnosis and management of rheumatoid arthritis. Am Fam Physician 2005; 72(6):10371047.

15. Gonzalez-Juanatey C, Llorca J, Testa A, Revuelta J, GarciaPorrua C, Gonzalez-Gay M. Increased prevalence of severe subclinical atherosclerotic findings in long-term treated rheumatoid arthritis patients without clinically evident atherosclerotic disease. Medicine (Baltimore) 2003; 82:40713

16. Stavropoulos-Kalinoglou A, Metsios G, Panoulas $V$ et al. Associations of obesity with modifiable risk factors for the development of cardiovascular disease in patients with rheumatoid arthritis. Annals of the Rheumatic Diseases. 2009; 68(2):242-245.

17. Panoulas V, Metsios G, Pace A et al. Hypertension in rheumatoid arthritis. Rheumatology. 2008:47(9):1286- 1298

18. Pereira R, de Carvalho J, Bonf'a E. Metabolic syndrome in rheumatological diseases. Autoimmunity Reviews 2009;8(5): 415-419.

19. Kitas G, Gabriel S. Cardiovascular disease in rheumatoid arthritis: state of the art and future perspectives. Annals of the Rheumatic Diseases.2011; 70:8-14.

20. Myasoedova E, Crowson C, Kremers H, Roger V, FitzGibbon P, Therneau $\mathrm{T}$ et al. Lipid paradox in rheumatoid arthritis: the impact of serum lipid measures and systemic inflammation on the risk of cardiovascular disease. Ann Rheum Dis 2011; 70:482-487.

21. Ku I, Imboden J, Hsue P, Ganz P. Rheumatoid arthritis-a model of systemic inflammation driving atherosclerosis. Circulation Journal. 2009; 73(6):977- 985.

22. Gonzalez-Gay M, Gonzalez-Juanatey C, Martin J. Rheumatoid arthritis: a disease associated with accelerated atherogenesis. Seminars in Arthritis and Rheumatism. 2005; 35(1):8-17.

23. Myasoedova E, Gabriel S. Cardiovascular disease in rheumatoid arthritis: a step forward. Current Opinion in Rheumatology. 2010; 22 (3):342-347

24. Kim S, Lee C, Lee E, Park S, Cho Y, Yoo B et al. Serum oxidized low density lipoproteins in rheumatoid arthritis. Rheumatology International. 2004;24(4):230-233

25. Del Rincon I, Williams K, Stern M, Freeman G, Escalante A. High incidence of cardiovascular events in rheumatoid arthritis cohort not explained by traditional cardiac risk factors. Arthritis Rheum 2001; 44:2737-45.

26. Mirjafari H, Al-Husain A, Bruce I. Cardiovascular risk factors in inflammatory arthritis. Current Opinion in Lipidology. 2011; 22(4):296-301.

27. Szekanecz Z, Kerekes G, Dér H, Sándor Z, Szabó Z, Végvári $A$, et al. Accelerated aterosclerosis in rheumatoidarthritis. AnnNYAcadSci 2007; 1108:349-58.

28. Snow M, Mikuls T. Rheumatoid arthritis and cardiovascular disease: the role of systemic inflammation and evolving strategies of prevention. CurrOpinRheumatol 2005;17:23441.

29. Toms $T$, Panoulas $V$, Douglas $K$, Nightingale $P$, Smith J, Griffiths $\mathrm{H}$ et al. Are lipid ratios less susceptible to change with systemic inflammation than individual lipid components in patients with rheumatoid arthritis? Angiology 2011; 62(2):167-175

30. Dessein P, Joffe B, Stanwix A, Botha A, Moomal Z. The acute phase response does not fully predict the presence of insulin resistance and dyslipidaemia in inflammatory arthritis. J Rheumatol 2002; 29: 462-6

31. Peters MJ, Vis M, van Halm VP, et al. Changes in lipid profile during infliximab and corticosteroid treatment in rheumatoid arthritis. Ann Rheum Dis 2007; 66: 958-61.

32. Choy E, Sattar N. Interpreting lipid levels in the context of high-grade inflammatory states with a focus on rheumatoid arthritis: a challenge to conventional cardiovascular risk actions. Ann Rheum Dis 2009;68:460-46

33. Situnayake R, Kitas G. Dyslipidemia and rheumatoid arthritis. Ann Rheum Dis 1997; 56:341-342

34. Chobanian AV, Bakris GL, Black HR, Cushman WC, Green LA, Izzo JL et al. The Seventh Report of the Joint National Committee on Prevention, Detection, Evaluation, and Treatment of High Blood Pressure: the JNC 7 report. JAMA. 2003;289:2560-72

35. American Diabetes Association. Standards of medical care in diabetes-2011: Position Statement Diabetes Care. 2011; 34(1):S11-61.

36. Ustrell-Roig $\mathrm{X}$ et al. Ictus. Diagnóstico y tratamiento de las enfermedades cerebrovasculares Rev Esp Cardiol. 2007;60(7):753-69

37. Thygesen et al. JACC Universal Definition of Myocardial Infarction. ESC/ACCF/AHA/WHF Expert Consensus Document 2007;50 (22):2173-95

38. Jimenez Ruiz CA, et al. Recomendaciones en el abordaje diagnóstico y terapéutico del tabaquismo. Documento de consenso Arch Bronconeumol 2003;39(1):35-41

39. Grundy S, Cleeman J, Bairey Merz H, Bryan Brewer C, Clark L, Hunninghake $D$ et al. Implications of recent clinical trials for the National Cholesterol Education Program ATP III guidelines. Circulation 2004; 110(2):227-239

40. World Health Organization. Obesity. 2008 [Accessed October 22, 2009]. Available at: http://www.who.int/topics/obesity/en/ 
41. Aletaha D, Ward M M, Machold KP, NellVP. Remission and active disease in rheumatoid arthritis: defining criteria for disease activity states. Arthritis Rheum 2005; 52:26252636

42. Anderson J, Zimmerman L, Caplan L, Michaud K. Measures of Rheumatoid Arthritis Disease Activity Arthritis Care \& Research, 2011; 63(S11): S14-S36

43. Georgiadis et al. Atherogenic lipid profile is a feature characteristic of patients with early rheumatoid arthritis: effect of early treatment - a prospective, controlled study. Arthritis Research \& Therapy 2006;8:R82

44. Dessein PH, Stanwix AE, Joffe BI: Cardiovascular risk in rheumatoidarthritis versus osteoarthritis: acute phase response related decreased insulin sensitivity and highdensity lipoproteincholesterol as well as clustering of metabolic syndrome features in rheumatoid arthritis. Arthritis Res 2002;4:R5.

45. Munro R, Morrison E, McDonald AG, Hunter JA, Madhok R, Capell HA: Effect of disease modifying agents on the lipid profiles of patients with rheumatoid arthritis. Ann Rheum Dis 1997; 56:374-377.

46. Full, L. et al .The inextricable link between atherosclerosis and prototypical inflammatory diseases rheumatoid arthritis and systemic lupus erythematosus. Arthritis Research \& Therapy 2009;11:217

47. Galindo J. et al. Dyslipidemia in patients with rheumatoid arthritis attended at a general hospital. Rev Med Hered 2011;22:47-53

48. Boers M, Nurmohamed M, Doelman C, Lard L, Verhoeven A, Voskuyl A et al. Influence of glucocorticoid and disease activity on total and high density lipoprotein cholesterol in patients with rheumatoid arthritis. Ann Rheum Dis 2003;62:842-845

49. Park Y, Lee S, Lee W, Suh C, Lee C, Lee C et al. Lipid profiles in untreated patients with rheumatoid arthritis. J Rheumatol 1999;26:1701-1704
50. Vibhuti Singh.Low high-density lipoprotein cholesterol: current status and future strategies for management Vascular Health and Risk Management 2010;6: 979-996

51. Park Y.; Choi, $\mathrm{H}$ et al. Effects of antirheumatic therapy on serum lipid levels in patients with rheumatoid arthritis: a prospective study The American Journal of Medicine 2002; 113(3):188-193

52. Lorber M, Aviram M, Linn S, Scharf $Y$, Brook JG: Hypocholesterolaemia and abnormal high-density lipoprotein in rheumatoid arthritis. Br J Rheumatol 1985; 24:250-255.

53. Frati E, Castagna ML, Bacarelli MR, Fioravanti A, Giordano $\mathrm{N}$, Taddeo A et al. Plasma levels of apolipoprotein and HDLcholesterol in patients with rheumatoid arthritis. Boll SocltalBiolSper1984;60:1791-1796

54. Choi H, Seerger J. Lipid Profiles Among US Elderly with Untreated Rheumatoid Arthritis - The Third National Health and Nutrition Examination Survey. (NHANES III) J Rheumatol 2005;32:2311-6

55. Naranjo, A et al. Research article Cardiovascular disease in patients with rheumatoid arthritis: results from the QUESTRA study, Arthritis Research \& Therapy 2008, 10:R30

56. van Halm V, Nielen M, Nurmohamed M, van Schaardenburg D, Reesink H., Voskuyl E. et al. Lipids and inflammation: serial measurements of the lipid profile of blood donors who later developed rheumatoid arthritis. Ann Rheum Dis 2007; 66:184-188.

57. Myasoedova E. et al. Total Cholesterol and LDL levels decrease before rheumatoid arthritis Ann Rheum Dis. 2010 July ; 69(7): 1310-1314.

58. Svenson KLG, Lithell $H$, Hällgren, et al. Serum lipoprotein in activerheumatoid arthritis and other chronic infl ammatory arthritides.I Relativity to infl ammatory activity. Arch Intern Med, 1987; 147:1912-16

59. White D, Fayez S, Doube A. Atherogenic lipid profi les in rheumatoid arthritis. N Z Med 2006; 119:U2125 